\title{
Distribution of Acoustic Power Spectra for an Isolated Helicopter Fuselage
}

\author{
A.N. Kusyumov ${ }^{1, a}$, S.A. Mikhailov ${ }^{2}$, L.I. Garipova ${ }^{3}$, A.S. Batrakov ${ }^{4}$ and G. Barakos ${ }^{5}$ \\ ${ }^{1-4}$ Kazan National Research Technical University n.a. A. N. Tupolev (KNRTU-KAI), Kazan, Russia \\ ${ }^{5}$ University of Liverpool, Liverpool, UK
}

\begin{abstract}
The broadband aerodynamic noise can be studied, assuming isotropic flow, turbulence and decay. Proudman's approach allows practical calculations of noise based on CFD solutions of RANS or URANS equations at the stage of post processing and analysis of the solution. Another aspect is the broadband acoustic spectrum and the distribution of acoustic power over a range of frequencies. The acoustic energy spectrum distribution in isotropic turbulence is non monotonic and has a maximum at a certain value of Strouhal number. In the present work the value of acoustic power peak frequency is determined using a prescribed form of acoustic energy spectrum distribution presented in papers by S. Sarkar and M. Y. Hussaini and by G. M. Lilley. CFD modelling of the flow around isolated helicopter fuselage model was considered using the HMB CFD code and the RANS equations.
\end{abstract}

\section{Introduction}

For a conventional helicopter, there are two fundamental elements that contribute to the generation of near-field and far-field noise, the main rotor and the tail rotor [1]. Engine and fuselage noise are typically of secondary significance. A helicopter main rotor generates primarily tonal and broadband noise. Additional sources, including Blade Vortex Interaction (BVI) noise and High Speed Impulsive (HSI) noise, that are dominant at specific flight regimes. Basic loading noise during hover is generally dominant in a conical region directed 30 to 40 degrees downward from the rotor plane, while broad-band noise radiates mostly out of the plane of the rotor [1]. So for some operating conditions and for specific directions of sound propagation, the broadband noise generated by all parts of helicopter (including fuselage) can be a significant contributor to the overall helicopter noise.

According to Proudman [2] the broadband noise can be considered, assuming isotropic flow turbulence and decay. The total acoustic power radiated from embedded finite regions of turbulence contained within an infinite volume of compressible fluid, is a function of the local time-averaged kinetic energy of the turbulence per unit volume, $\mathrm{k}$, and the time averaged rate of dissipation of the kinetic energy per unit volume, $\varepsilon$.

Another aspect, is the broadband acoustic spectrum and the distribution of acoustic power over a range of frequencies. The acoustic energy spectrum distribution in isotropic turbulence is non monotonic. The power spectrum function has a maximum at some value of Strouhal number. In the present work the value of acoustic power peak frequency is determined using a prescribed form of acoustic energy spectrum distribution presented in papers by S. Sarkar and M. Y. Hussaini [3] and by G. M. Lilley [4].

CFD modelling of the flow around isolated helicopter fuselage model is considered in this paper using the HMB CFD code and the RANS equations.

\section{Broadband isotropic turbulence noise (Proudman's formula)}

A simple approach to estimate acoustic emission of flying vehicle for turbulent flows, assumes that the emitted noise does not have any distinct tones, and that the sound energy is continuously distributed over a broad range of frequencies. In this case the broadband noise power, can be estimated from RANS equations using the mean flow

\footnotetext{
${ }^{1 \mathrm{a}}$ Prof., department of aero-hydrodynamics, postbox7@mail.ru

${ }^{2}$ Prof., department of aero-hydrodynamics, sergey.mikhaylov@kai.ru

${ }^{3} \mathrm{PhD}$ student, lyaysan_garipova@mail.ru

${ }^{4} \mathrm{PhD}$ student, batrakov_a.c@mail.ru

${ }^{5}$ Prof., department of Engineering, George.Barakos@glasgow.ac.uk
} 
field, turbulent kinetic energy $\mathrm{k}$ and the dissipation rate $\varepsilon$. Unlike the direct method of simulation and the integral methods, Proudman's [2] approach does not require unsteady CFD solutions and is based on Lighthill's acoustic analogy [5].

The intensity $I(\mathbf{x}, t)$ of the radiated sound in the farfield is proportional to the square of the fluctuating pressure $p$ and is defined by

$$
I(\mathbf{x}, t)=\frac{<\left(p-p_{\infty}\right)^{2}>}{\rho_{\infty} c_{\infty}},
$$

were $\rho_{\infty}, p_{\infty}$ are the air density and pressure, $c_{\infty}$ is the ambient sound speed. The intensity of radiated sound at any point $\mathbf{x}$, and at time $t$ can be determined as follows $[4,6]$ :

$I(\mathbf{x}, t)=\frac{1}{16 \pi^{2} \rho_{\infty} c_{\infty}^{5} x^{2}} \iiint d \mathbf{y} \iiint d \boldsymbol{r} \frac{\partial^{4}}{\partial \tau^{4}} P_{x x, x x}(\mathbf{y}, \mathbf{r}, \tau)$.

Here $\rho_{\infty}$ is the air density, $c_{\infty}$ is the ambient sound speed, $\mathbf{y}$ is a position, $\mathbf{r}$ is the distance vector between two internal points $\mathbf{y}$ and $\mathbf{y}^{\prime}$ inside the integration volume, and $\tau$ is the retarded time, $x=|\mathbf{x}|$. The space-time correlation function $P_{x x, x x}$ can be written in the form

$$
P_{x x, x x}=\alpha_{P} u^{4} \Phi(r / l) \Psi(\Omega \tau)
$$

with the non-dimensional space correlation function, $\Phi(r / l)$, and the non-dimensional retarded time correlation function, $\Psi(\Omega \tau)$. Here $u, l$, and $\Omega$ are, respectively, reference values of the turbulent velocity, the turbulent length scale, and turbulent frequency; $\alpha_{P}=$ const is a scale coefficient $\left(\alpha_{P}=1\right.$ in [4]). The forms for the space and temporal correlation functions accepted in [4] are

$$
\Phi(r / l)=\exp \left(-\frac{\pi}{2} \frac{r^{2}}{L^{2}}\right), \Psi(\Omega \tau)=\exp \left(-\frac{\pi}{2} \Omega^{2} \tau^{2}\right),
$$

where $L$ is the integral lenght scale.

Using the expression (2) for the space correlation function, Proudman obtained an estimate of the acoustic power per unit of volume in the time domain:

$$
P_{a t}=\alpha_{\varepsilon} \rho_{\infty} \varepsilon M_{t}^{5},
$$

where

$$
M_{t}=(2 k)^{1 / 2} / c_{\infty}
$$

is the turbulent Mach number, $k$ is the is turbulent kinetic energy per unit mass, and $c_{\infty}$ is the speed of sound. The turbulent dissipation rate $\varepsilon$ can be determined [7] by

$$
\varepsilon=\frac{k^{3 / 2}}{L} \text {. }
$$

Sarkar and Hussaini [3] recommended the re-scaled constant, $\alpha_{\varepsilon}=0.1$, based on DNS data. It should be noted that there are different expressions for the reference turbulent velocity $u$ (see, for example, paper [2], were $\left.u=(2 k / 3)^{1 / 2}\right)$. In this paper $u=(2 k)^{1 / 2}$ following reference [8].

\section{Total radiated acoustic power}

An alternative approach to determine the acoustic power per unit of volume is based on the spectral transformation of the intensity $I(\mathbf{x}, t)$ of the radiated sound. The spectral density $I(\mathbf{x}, \omega)$ corresponding to the intensity $I(\mathbf{x}, t)$ of the radiated sound is $([6])$

$$
\begin{gathered}
I(\mathbf{x}, \widetilde{\omega})=\frac{1}{2 \pi} \int I(\mathbf{x}, t) \exp (i \widetilde{\omega} t) d t= \\
=\frac{\pi \omega^{4}}{2 x^{2} \rho_{\infty} c_{\infty}^{5}} \int_{V} P_{x x, x x}(\mathbf{y}, \mathbf{k}, \widetilde{\omega}) d^{3} \mathbf{y} .
\end{gathered}
$$

The frequency of the sound $\widetilde{\omega}$ is the same as in the turbulence and $\mathbf{k}$ is the wavenumber vector of the sound. Taking into account (2) the space-time correlation function (1) may be written:

$$
P_{x x, x x}=\alpha_{P} u^{4} \exp \left(-\frac{\pi}{2}\left(\frac{r^{2}}{L^{2}}+\Omega^{2} \tau^{2}\right)\right)
$$

with four-dimensional wave-number/frequency spectrum function [4]:

$$
P(k, \widetilde{\omega})=\frac{1}{4 \pi^{4}} \frac{u^{7} S_{T}^{3}}{\Omega^{4}} \exp \left(-\frac{\widetilde{\omega}^{2}}{2 \pi \Omega^{2}} C\right) .
$$

and the acoustic spectral density

$$
P_{s}(\widetilde{\omega})=2 \pi^{2} \frac{\rho_{\infty}}{c_{\infty}^{5}} \omega^{4} P(k, \widetilde{\omega}) .
$$

Here

$$
S_{T}=\Omega L / u
$$

is the turbulent Strouhal number and

$$
C=1+S_{T}^{2} u^{2} / c_{\infty}^{2}=1+S_{T}^{2} M_{t}^{2} .
$$

According to [4] the total radiated acoustic power per unit volume of turbulence is in the frequency domain:

$$
P_{a \omega}=\alpha_{P} \int_{0}^{\infty} P_{S}(\widetilde{\omega}) d \widetilde{\omega} .
$$

Substituting (5), (6) gives the total radiated acoustic power per unit volume of turbulence

$$
P_{a \omega}=\alpha_{P} \rho_{\infty} \frac{3 \pi}{\sqrt{2} C^{5 / 2}} \frac{u^{3}}{L} S_{T}^{4} M_{t}^{5} .
$$

\section{Proudman's constant}

The expression (3) for the total acoustic power radiated per unit volume of flow in the time domain can be written as

$$
P_{a t}=\alpha \rho_{\infty} \frac{u^{3}}{L} M_{t}^{5},
$$

where $\alpha$ is Proudman's constant. Taking into account (4) the re-scaled constant $\alpha_{\varepsilon}$, and Proudman's constant are connected by the expression:

$$
\alpha_{\varepsilon}=(2)^{3 / 2} \alpha \text {. }
$$

The proposal that the total acoustic power radiated per unit volume in the time domain (3) is equal to the acoustic power in the frequency domain (9) can be written as

$$
P_{a t}=P_{a \omega} \text {. }
$$

After substitution (3) and (8) to (11) we have

$$
\alpha=\alpha_{P} \frac{3 \pi}{\sqrt{2} C^{5 / 2}} S_{T}^{4} \text {. }
$$

In this case, taking into account (10), we can rewrite Proudman's formula (3) in the form

$$
P_{a t}=\alpha_{P} \frac{6 \pi}{C^{5 / 2}} S_{T}^{4} \rho \varepsilon M_{t}^{5}
$$

According to the approximate estimations [4] the turbulent Strouhal number can be accepted as

$$
S_{T} \sim 1 \text {. }
$$

For low speed subsonic flow $\left(M_{t}<<1\right.$ or $\left.C \approx 1\right)$ we have the approximate value

$$
\alpha_{P} \approx \alpha \frac{\sqrt{2}}{3 \pi}=\frac{\alpha_{\varepsilon}}{6 \pi}=0.0053 .
$$




\section{Turbulent reference frequency}

The condition (13) and expression (4) can be used to determine the reference frequency $\Omega$

$$
\Omega \approx u / L=\sqrt{2} \varepsilon / k .
$$

As $\varepsilon=\beta^{*} k \omega(\omega$ is the specific dissipation parameter, $\left.\beta^{*}=0.09\right)[8]$ we finally have

$$
\Omega \approx \sqrt{2} \beta^{*} \omega .
$$

According to [4] the reference frequency $\Omega$ can be used to determine a frequency peak in the acoustic power spectrum:

$$
\omega_{m}=\sqrt{8} \Omega
$$

and with formula (14) we can rewrite (15) as

$$
\omega_{m} \approx 4 \beta^{*} \omega \text {. }
$$

Expression (3) can be rewritten in the form

$$
P_{a t}=\frac{1}{4} \alpha_{\varepsilon} \rho_{\infty} c_{\infty}^{2} \beta^{*} \omega M_{t}^{7}
$$

or

$$
P_{a t}=\frac{1}{16} \alpha_{\varepsilon} \rho_{\infty} c_{\infty}^{2} \omega_{m} M_{t}^{7} \text {. }
$$

Formulas (16) - (18) allow estimation of the frequency peak and the acoustic power per unit volume spatial the steady computation of the flow using the turbulent Mach number $M_{t}$ and the specific dissipation $\omega$ for the k- $\omega$ turbulence model [9].

Another estimation of the acoustic power and the acoustic intensity was given by Lighthill [5]:

$$
\begin{array}{r}
P_{a} \sim \frac{\rho_{\infty} u^{8}}{c_{\infty}^{5}} L_{r e f}^{2}, \\
I \sim \frac{\rho_{\infty} u^{8}}{c_{\infty}^{5}} .
\end{array}
$$

Here $L_{r e f}$ is a reference length. From the comparison of expressions (19) and (20) we have that

$$
I \sim \frac{P_{a}}{L_{r e f}^{2}} \text {. }
$$

Eventually we can write for the local sound pressure at the vicinity of an emitting body surface:

$$
p_{s}=\left(I \rho_{\infty} c_{\infty}\right)^{1 / 2}=\left(\frac{P_{a} \rho_{\infty} c_{\infty}}{L_{r e f}^{2}}\right)^{1 / 2} .
$$

\section{Numerical simulation of flow around an isolated helicopter fuselage}

For simulations, an early version of the ANSAT helicopter produced by the JSC Kazan Helicopters is used. CFD computations and experiments were conducted for fuselage model with (without) skids and cross-beams. Multiblock grids for the CFD computations were constructed using the ANSYS ICEM-hexa software. The computational domain was resolved using hexahedral grids and the 3D steady incompressible Reynolds-Averaged Navier-Stokes (RANS) equations. Fully turbulent calculations were performed using the $k-\omega$ model [9]. The computational hexa-grid for this model (without skids and cross-beams) contained 964 blocks and about 13.500 .000 cells. The computations were performed using the HMB solver of Liverpool University
[10]. The results of the CFD modelling were compared to wind tunnel experimental data. Fuselage was tested at the low speed T-1K wind tunnel of KNRTU-KAI $(2.25 \mathrm{~m}$ diameter).

The wind tunnel model fuselage was constructed using the same CAD model (figure 1), used for CFD modelling. The mesh topology and the surface grid near the area of the exhausts are presented in figure 2 . The aerodynamic performances of this model and CFD code validation were considered in references [11-14], and were studied using the $\mathrm{T}-1 \mathrm{~K}$ wind tunnel, that is equipped with a six-component Prandtl-type balances. The conditions of the wind tunnel experiment and CFD modelling corresponded to the free stream Mach number and the Reynolds numbers were of 0.1 and $4.4 \cdot 10^{6}$, respectively.

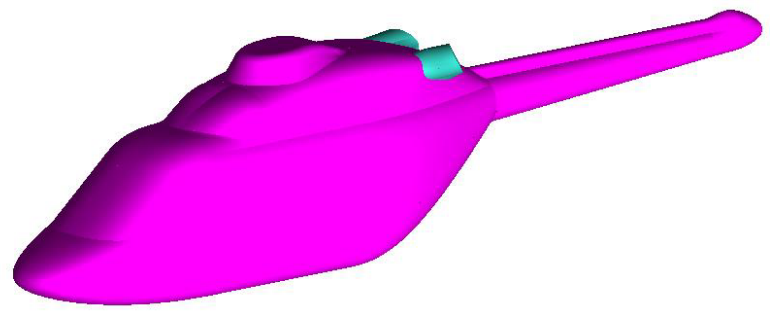

Fig. 1. Isolated helicopter fuselage model.
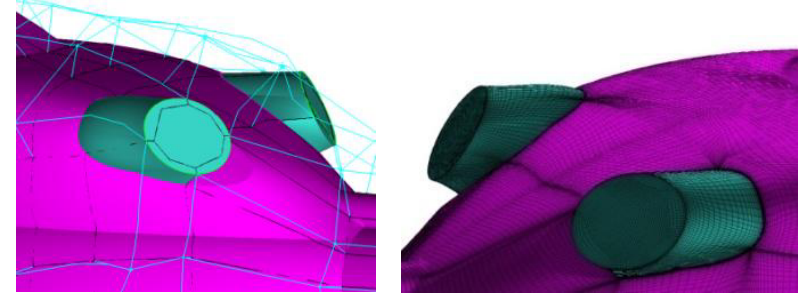

Fig. 2. Topology of blocks, and surface grid at the area of the engine exhausts.

Results of numerical simulation for the value of pitch angle of 0 degrees, including visualization of the Sound Pressure Level, the vector field and the frequency peak in the acoustic power spectrum are resented at sections of flow, according to the slices map in figure 3 .

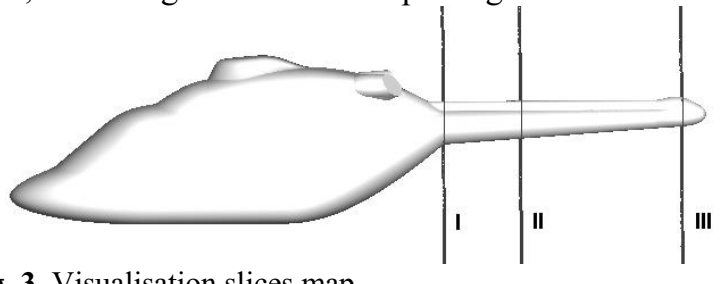

Fig. 3. Visualisation slices map.

The velocity field distribution scale is estimated according to the expression

$$
\bar{V}=\frac{V}{V_{\infty}},
$$

were $V$ is a local velocity value, $V_{\infty}$ is the free stream velocity. The Sound Pressure Level (SPL, dB) is evaluated according the formula

$$
\mathrm{SPL}=20 \log _{10}\left(p_{s} / p_{\text {ref }}\right) \text {. }
$$

Here $p_{s}$ is the sound pressure, according to equations (21), and $p_{\text {ref }}=2 \times 10^{-5} \mathrm{~Pa}$. 
2D distribution of velocity field distributions and flow streamlines (figure 4) are given in the sections of the slices map. The vortex structures visualized at the cross flow sections are generated by the exhausts, the engine faring and the rear part of fuselage.

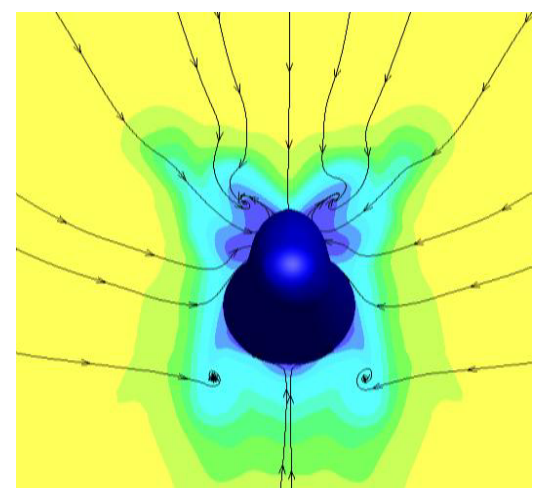

a)

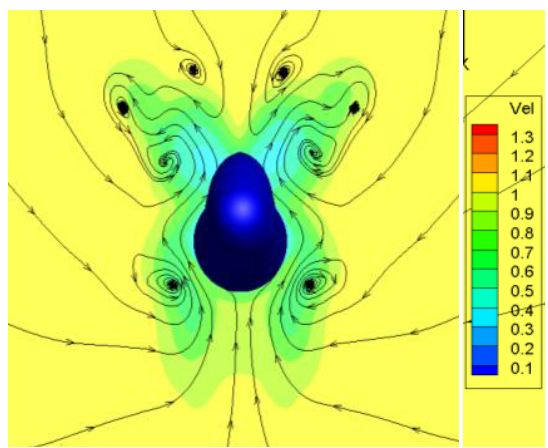

b)

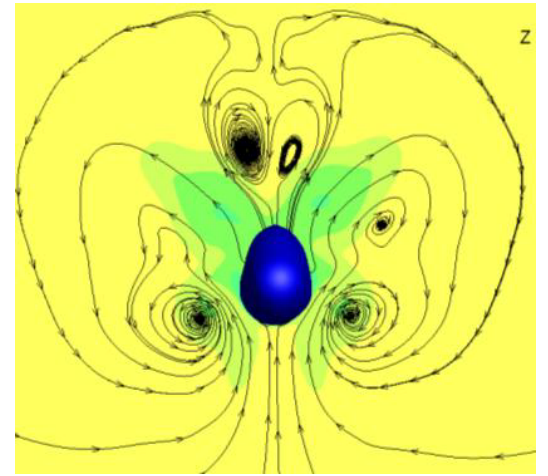

c)

Fig. 4. Velocity field distributions and flow streamlines for the sections a) I, b) II, c) III.

Localisation of the vortex structures in general corresponds to perturbations of the velocity field in figure 4. It can be expected that perturbations of the velocity field area correspond to the distribution of high SPL intensity, presented in figure 5.

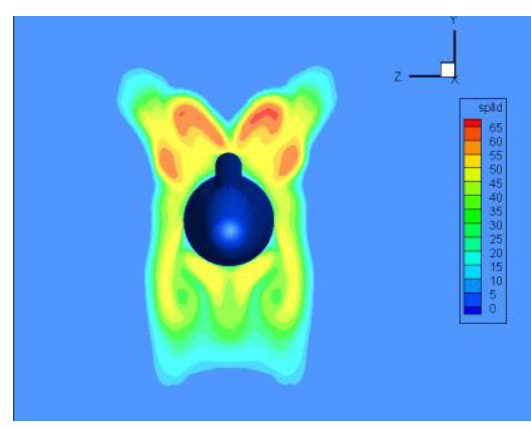

a)

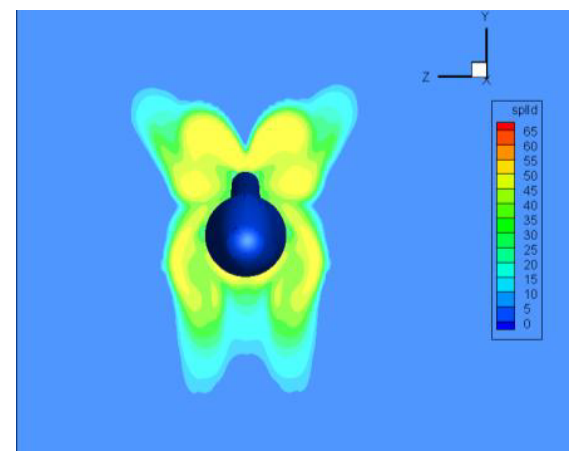

b)

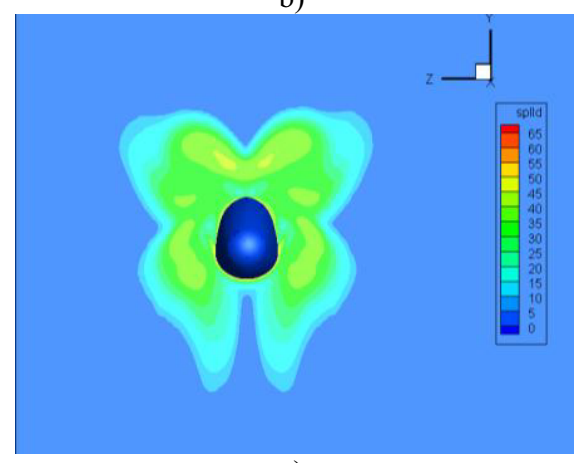

c)

Fig. 5. SPL distributions for the sections a) I, b) II, c) III.

Figure 6 presents the distribution of frequency peak in the acoustic power spectrum distribution (see equation (16)) for the same sections. Lowering of the vector field disturbances (figure 4) leads to lowering of the SPL intensity (figure 5) and to decreasing of the frequencies spectrum area (figure 6).

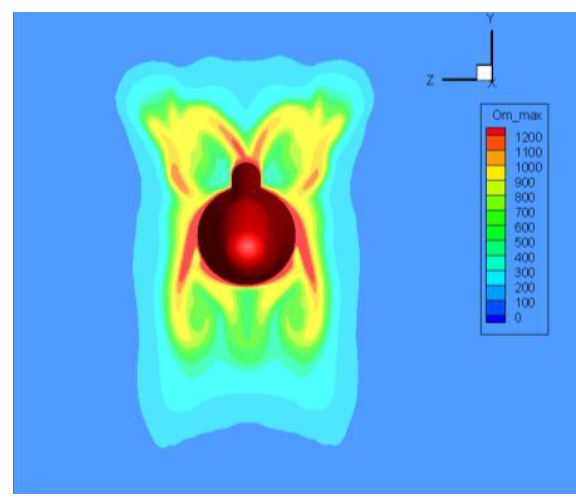

a) 


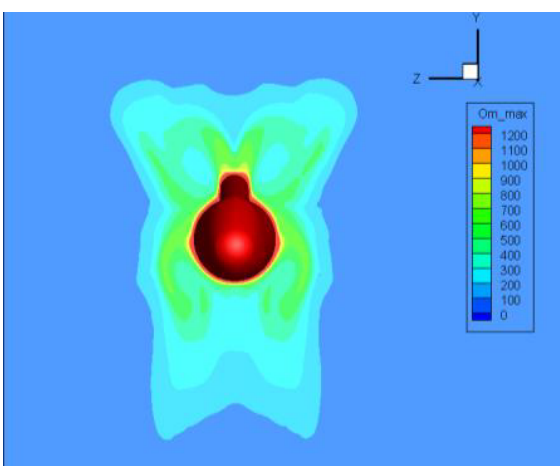

b)

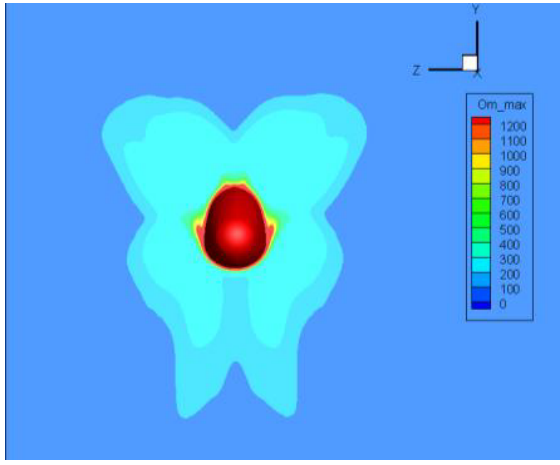

c)

Fig. 6. The frequency peak in the acoustic power spectrum distribution for the sections a) I, b) II, c) III.

\section{Conclusions and future work}

The acoustic properties (broadband noise) of the flow, and the frequency peak in the acoustic power spectrum distribution were estimated. Using Lighthill's acoustic analogy and the RANS equations, the broadband noise Sound Pressure Level was estimated based on the Proudman's formula. Proudman's approach was combined with Lilley's estimations to determine the frequency of the acoustic power spectrum distribution peak distribution. The structure and the acoustic properties of the flow around an isolated fuselage helicopter were examined in the in terms of mean flow field, turbulent kinetic energy and the dissipation rate.

In the future, the problem of the helicopter fuselage acoustic properties will be considered using CFD modelling based on the URANS equations and advances turbulence models.

This work is supported by the "Project part of state task in the field of the scientific activity" Grant (No 9.1694.2014/K).

\section{References}

1. Ch. Edwards Band Cox, NASA/CR-2002-211650, May (2002).

2. I. Proudman, Proc. Roy. Soc., 214, 119-132, August (1952).
3. S. Sarkar, M. Hussaini, ICASE Rep, 93-74 (1993).

4. G. Lilley, NASA Contract Report, 93-75, NASA Langley Research Center, Hampton, VA (1993).

5. M. J. Lighthill, Proc. Roy. Soc. London Ser, 211, 564 587. MR 13,879i. Zbl 049. 259 (1952).

6. G. Lilley, Center for Turbulence Research, Annual Research Briefs, 241 - 255 (1995).

7. H. K. Versteeg and W. Malalasekera, Pearson Education Limited, 503, (2007).

8. ANSYS FLUENT 12.0/12.1 Documentation.

9. D. C. Wilcox, AIAA J., 26, 11, 1299-1310 (1988).

10. G. Barakos, R. Steijl, K. Badcock and A. Brocklehurst, 31st European Rotorcraft Forum, 13-15 September 2005, Florence, Italy (2005).

11. A. Kusyumov, S. Mikhailov, E. Romanova, A. Garipov, E. Nikolaev, G. Barakos, EFM 2012 conference, Hradec Kralove, Czech. Republic (2012).

12. A. Batrakov, A. Kusyumov, S. Mikhailov, V. Pakhov, A. Sungatullin, V. Zherekhov, G. Barakos, 39 European Rotorcraft Forum, Moscow, Russia (2013).

13. A. Kusyumov, S. Mikhailov, A. Garipov, E. Nikolaev, G. Barakos, Transactions on Control and Mechanical Systems, 1, 7, 318 - 324 (2012).

14. A. Batrakov, A. Kusyumov, S. Mikhailov, V. Pakhov, A. Sungatullin, V. Zherekhov, G. Barakos, ASME 2013 International Mechanical Engineering Congress, San Diego, California, USA (2013). 